OPEN ACCESS

Edited by:

Bart Spronck,

Maastricht University, Netherlands

Reviewed by:

Alessandro Giudici,

Maastricht University, Netherlands

Carlo Palombo,

University of Pisa, Italy

*Correspondence:

Mohsen Agharazi

mohsen.agharazii@

crchudequebec.ulaval.ca

${ }^{\dagger}$ These authors have contributed equally to this work and share first

authorship

Specialty section

This article was submitted to

Vascular Physiology,

a section of the journal

Frontiers in Physiology

Received: 10 September 2021

Accepted: 12 November 2021

Published: 15 December 2021

Citation:

Fortier C, Garneau C-A, Paré M Obeid H, Côté N, Duval K, Goupil R and Agharazii M (2021) Modulation of Arterial Stiffness Gradient by Acute

Administration of Nitroglycerin.

Front. Physiol. 12:774056.

doi: 10.3389/fphys.2021.774056

\section{Modulation of Arterial Stiffness Gradient by Acute Administration of Nitroglycerin}

\author{
Catherine Fortier ${ }^{1,2,3 \dagger}$, Charles-Antoine Garneau'1,2†, Mathilde Paré1,2, Hasan Obeid ${ }^{1}$, \\ Nadège Côté1,2, Karine Duval' ${ }^{1}$, Rémi Goupil ${ }^{3}$ and Mohsen Agharazii1,2*
}

${ }^{1}$ CHU de Québec Research Center-Université Laval, L'Hôtel-Dieu de Québec Hospital, Québec, QC, Canada, ${ }^{2}$ Division of Nephrology, Faculty of Medicine, Université Laval, Québec, QC, Canada, ${ }^{3}$ Research Center of the Hôpital du Sacré-Coeur de Montréal, Montréal, QC, Canada

Background: Physiologically, the aorta is less stiff than peripheral conductive arteries, creating an arterial stiffness gradient, protecting microcirculation from high pulsatile pressure. However, the pharmacological manipulation of arterial stiffness gradient has not been thoroughly investigated. We hypothesized that acute administration of nitroglycerin (NTG) may alter the arterial stiffness gradient through a more significant effect on the regional stiffness of medium-sized muscular arteries, as measured by pulse wave velocity (PWV). The aim of this study was to examine the differential impact of NTG on regional stiffness, and arterial stiffness gradient as measured by the aortic-brachial PWV ratio (AB-PWV ratio) and aortic-femoral PWV ratio (AF-PWV ratio).

Methods: In 93 subjects (age: 61 years, men: 67\%, chronic kidney disease [CKD]: $41 \%)$, aortic, brachial, and femoral stiffnesses were determined by cf-PWV, carotidradial (cr-PWV), and femoral-dorsalis pedis artery (fp-PWV) PWVs, respectively. The measurements were repeated 5 min after the sublingual administration of NTG (0.4 mg). The AB-PWV and AF-PWV ratios were obtained by dividing cf-PWV by cr-PWV or fp-PWV, respectively. The central pulse wave profile was determined by radial artery tonometry through the generalized transfer function.

Results: At baseline, cf-PWV, cr-PWV, and fp-PWV were $12.12 \pm 3.36,9.51 \pm 1.81$, and $9.71 \pm 1.89 \mathrm{~m} / \mathrm{s}$, respectively. After the administration of NTG, there was a significant reduction in cr-PWV of $0.86 \pm 1.27 \mathrm{~m} / \mathrm{s}(p<0.001)$ and fp-PWV of $1.12 \pm 1.74 \mathrm{~m} / \mathrm{s}(p<0.001)$, without any significant changes in cf-PWV $(p=0.928)$, leading to a significant increase in the AB-PWV ratio (1.30 \pm 0.39 vs. $1.42 \pm 0.46$; $p=0.001)$ and AF-PWV ratio (1.38 \pm 0.47 vs. $1.56 \pm 0.53 ; p=0.001)$. There was a significant correlation between changes in the AF-PWV ratio and changes in the timing of wave reflection ( $r=0.289 ; p=0.042)$ and the amplitude of the heart rate-adjusted augmented pressure $(r=-0.467 ; p<0.001)$. 
Conclusion: This study shows that acute administration of NTG reduces PWV of muscular arteries (brachial and femoral) without modifying aortic PWV. This results in an unfavorable profile of AB-PWV and AF-PWV ratios, which could lead to higher pulse pressure transmission into the microcirculation.

Keywords: arterial stiffness gradient, arterial stiffness, aortic stiffness, pulse wave velocity (PWV), nitroglycerin (NTG), pulse wave analysis (PWA), chronic kidney disease (CKD), arterial compliance

\section{INTRODUCTION}

Aortic stiffness, determined by aortic pulse wave velocity (PWV), is a hallmark of vascular aging and an independent cardiovascular risk factor in various clinical conditions ranging from hypertension to end-stage kidney disease (Blacher et al., 1999a,b; Laurent et al., 2001; Cruickshank et al., 2002; Karras et al., 2012). The consequences of aortic stiffening on the heart are essentially explained by an increased cardiac workload and a decreased coronary perfusion pressure. However, the peripheral target organ damage may best be explained by the arterial stiffness gradient hypothesis (Mitchell, 2008). Indeed, due to the heterogeneity of vascular wall composition and diameter, there is an increase in arterial stiffness (stiffness gradient) from the heart to the periphery. This gradient in arterial stiffness results in a gradual attenuation of forward pressure wave along the arterial tree down to the microcirculation, where the pulsatility must be minimal. In the process of vascular aging, medium-sized arteries show little changes compared to the aorta, leading to attenuation or even a reversal of the stiffness gradient (Mitchell, 2008; Fortier and Agharazii, 2016). As a consequence, a higher pulse pressure could be transmitted into the microcirculation, causing exaggerated vascular myogenic response, and ultimately hypoperfusion and organ damage (Mitchell, 2008; Fortier and Agharazii, 2016). Indeed, a number of recent studies have underlined the relevance of arterial stiffness gradient on both blood flow and clinical outcomes (Mitchell et al., 2011; Tarumi et al., 2011; Hashimoto and Ito, 2013).

In the context of chronic kidney disease (CKD), we have previously used the PWV ratio between cf-PWV and cr-PWV as a surrogate measure of arterial stiffness gradient and have shown that it was a better prognostic indicator than cf-PWV alone (Fortier et al., 2015). In CKD patients, we have shown that the changes in arterial stiffness gradient are not only guided by an increase in aortic stiffness but also by a decrease in the stiffness of the medium-sized muscular arteries such as the brachial artery (Utescu et al., 2013; Fortier et al., 2015). We have also observed that patients with chronic use of nitroglycerin (NTG), a vasodilatory drug used primarily to treat coronary artery disease and/or heart failure, had a higher PWV ratio. This observation led us to hypothesize that NTG may have a negative impact on arterial stiffness gradient given that it has a more pronounced effect on medium-sized conduit arteries where vascular smooth muscle cells are a major component of the arterial wall (Wilkinson et al., 2002; Pauca et al., 2005; Yamamoto et al., 2017). However, the pharmacological manipulation of arterial stiffness gradient has not been thoroughly studied.
Therefore, we aimed to examine the effect of acute administration of NTG on arterial stiffness gradient. Second, we aimed to study the effect of NTG on local stiffness parameters of the brachial artery and its relation to changes in cr-PWV. Finally, we investigated whether the vascular response to NTG is different in patients with CKD than subjects without a significant decline in renal function.

\section{MATERIALS AND METHODS}

\section{Study Design and Population}

This is an interventional study designed to examine the acute effects of NTG in a single session of hemodynamic assessment. The inclusion criteria were adult patients with stable blood pressure (BP) medication and without any acute episode of illness (infection or recent cardiovascular events). Patients were excluded if they had a history of adverse reactions to NTG or were on renal replacement therapy. The control group ( $n=55)$ was composed of apparently healthy subjects or hypertensive subjects with an estimated glomerular filtration rate $(e G F R) \geq 45 \mathrm{ml} / \mathrm{min} / 1.73 \mathrm{~m}^{2}$ [CKD-EPI equation (Levey et al., 2009)]. The CKD group $(n=38)$ was composed of patients with an eGFR $<45 \mathrm{ml} / \mathrm{min} / 1.73 \mathrm{~m}^{2}$ (i.e., Stage $3 \mathrm{~B}$ CKD and above) (Kidney Disease Improving Global Outcomes [KDIGO], 2013). This cutoff was used because subjects with an eGFR $\geq 45 \mathrm{ml} / \mathrm{min} / 1.73 \mathrm{~m}^{2}$ are not at substantially higher risk of cardiovascular disease (Padhy et al., 2017). The participants recruited were healthcare workers and patients treated in hypertension or CKD clinics of CHU de Québec-Université Laval hospital. Hypertension was defined as BP $\geq 140 / 90 \mathrm{mmHg}$ or the use of antihypertensive medications in patients with a history of hypertension. Cardiovascular disease was defined as myocardial infarction, coronary artery revascularization, or ischemic heart disease as shown by treadmill, echo, or thallium stress tests; stroke or lower extremity amputation; or revascularization. This protocol had been approved by the comité d'éthique de la recherche du CHU de Québec and was conducted in accordance with the Declaration of Helsinki. All procedures followed were in accordance with institutional guidelines, and each patient had provided informed written consent.

\section{Hemodynamic Measurements Sequence}

All measurements were performed after $15 \mathrm{~min}$ of rest in a supine position. Brachial artery BP was recorded using an automatic oscillometric sphygmomanometer BPM-100 (BP-Tru, Coquitlam, Canada) by an experienced operator who was 
present in the room. BP was recorded six times, with a 2 -min interval between each measurement, and the average of the last five measurements was used to determine the brachial systolic BP (SBP), diastolic BP (DBP), and brachial artery pulse pressure $(b \mathrm{PP})$. Immediately after BP measurements, radial pulse wave profile was recorded using applanation tonometry after calibration with brachial SBP and DBP (SphygmoCor system ${ }^{\circledR}$, AtCor Medical Pty. Ltd., Sydney, Australia), followed by the determination of PWVs in peripheral (brachial and femoral) and central (aortic) arterial segments using Complior ${ }^{\circledR}$ Analyse (Artech Medical, Pantin, France). Brachial BP, PWVs, and radial pulse wave profile measurements were repeated $5 \mathrm{~min}$ after the sublingual administration of $0.4 \mathrm{mg}$ of NTG with the same described procedures except that pulse wave profile has been taken once instead of thrice. Radial pulse wave profile post-NTG was introduced later in the measurement protocol and performed in the last 56 patients. Wall properties of the brachial artery were assessed in 57 subjects by a radiofrequency-based wall-tracking system (ART-LAB, Esaote, Maastricht, Netherlands) before and continuously during $10 \mathrm{~min}$ after NTG administration.

\section{Pulse Wave Velocity and Arterial Stiffness Gradients}

We determined PWVs by direct measurements of distance and using a maximal upstroke algorithm for pulse transit time. Carotid-radial PWV (cr-PWV, carotid-dorsalis pedis PWV (cp-PWV), and carotid-femoral PWV (cf-PWV were obtained on the same side, in triplicates. Femoral-dorsalis pedis PWV was calculated using the formula (cp distance - cf distance)/(cp transit time - cf transit time). Stiffness gradients from the aorta to the brachial artery (upper limb) and from the aorta to the femoral artery (lower limb) were obtained using the aortic-brachial PWV ratio (AB-PWV ratio $=c f-P W V / c r-P W V)$ and aortic-femoral $\mathrm{PWV}$ ratio $(\mathrm{AF}-$ $\mathrm{PWV}$ ratio $=\mathrm{cf}-\mathrm{PWV} / \mathrm{fp}-\mathrm{PWV})$, respectively.

\section{Local Brachial Stiffness Parameters}

With the right arm stabilized in an inflated cushion, pre-NTG end-diastolic diameter (d), intima-media thickness (IMT), and distension of the brachial artery $(\sim 3 \mathrm{~cm}$ above the elbow crease). The ART-LAB probe ( $10 \mathrm{MHz}$ linear probe) was fastened by a locking articulating arm mounted on a platform that allowed two-dimensional microadjustments and good stability for a 10 min-period of real-time measurements after sublingual NTG administration. Arterial wall properties were calculated as follows:

- Compliance $=\frac{\triangle A}{\triangle P}=\frac{A_{s}-A_{d}}{b P P}$, where $A_{s}$ and $A_{d}$ stand for the systolic and diastolic cross-sectional areas;

- Distensibility $(\mathrm{DC})=\frac{1}{A_{d}} \cdot \frac{\triangle A}{\triangle P}$;

- Luminal cross-sectional area (LCSA) $=\frac{\pi d^{2}}{4}$;
- Mean wall cross-sectional area (WCSA)

$$
=\pi\left(\frac{d+2 \times I M T}{2}\right)^{2}-\pi\left(\frac{d^{2}}{2}\right) ;
$$

- Incremental elastic modulus $\left(\mathrm{E}_{\mathrm{inc}}\right)=\frac{3}{D C}\left(1+\frac{L C S A}{W C S A}\right) ;(5)$

- Wall to lumen ratio $(\mathrm{W} /$ Lratio $)=2\left(\frac{I M T}{d}\right)$;

(Laurent et al., 1994, 2006; Bortolotto et al., 1999; Spronck et al., 2021).

The IMT measurements were not performed after NTG administration, but it was calculated based on the principle of WCSA conservation.

\section{Central Pulse Wave Profile Analysis}

Three consecutive recordings were performed on the radial artery to construct a central pulse wave profile using an integrated generalized transfer function. The average of central SBP, central DBP, central pulse pressure (PP), heart rate-adjusted central augmented pressure (AP@75), and augmentation index (AIx@75), as well as end systolic pressure, were measured. Period, ejection duration, diastolic duration, the first peak of pressure height (P1) with the respective time of each peak (T1, T2), time to the reflection of the reflected pressure wave (TR), and subendocardial viability ratio (SEVR) were also obtained (Miyashita, 2012).

\section{Statistical Analysis}

The results are expressed as mean $\pm \mathrm{SD}, n$ (\%), or median (25th75 th percentiles). Differences between groups were assessed using $t$-test, Mann-Whitney $U$-test, or chi-square accordingly. The effects of NTG on vascular parameters were examined using paired $t$-test. Linear regression was used to examine the relationship between renal function, PWVs, and PWV ratios. We used generalized estimating equations (GEE) to adjust changes in vascular parameters for changes in DBP. Linear regressions were performed to determine the relationship between cr-PWV and brachial artery diameter. GEEs were also used to compare if the vascular response to NTG was different between CKD and the control group. Forward conditional linear regression was used to identify the determinants of changes in cr-PWV after NTG administration. We used the Spearman rank correlation coefficient to assess the association between TR and AP@75 with both AB-PWV and AF-PWV ratios. Finally, as part of sensitivity analysis, we examined if there were any significant interactions between age, sex, and established cardiovascular disease, and the vascular response to NTG (GEE). All statistical analyses were performed using SPSS Statistics for Windows, Version 27.0 (IBM Corp., Armonk, NY, United States). A two-tailed $p$-value $<0.05$ was considered to be statistically significant.

\section{RESULTS}

\section{Patient Population}

Table 1 shows the baseline demographic, clinical, pharmacological, and biological characteristics of patients 
TABLE 1 | Characteristics of patients for control group, CKD group, and overall.

\begin{tabular}{|c|c|c|c|}
\hline & Overall & Control & CKD \\
\hline & & $\begin{array}{c}\text { eGFR } \geq \\
45 \mathrm{ml} / \mathrm{min} / 1.73 \mathrm{~m}^{2}\end{array}$ & $\begin{array}{c}\text { eGFR }< \\
45 \mathrm{ml} / \mathrm{min} / 1.73 \mathrm{~m}^{2}\end{array}$ \\
\hline Parameters & $n=93$ & $n=55$ & $n=38$ \\
\hline Male & $62(67)$ & $35(64)$ & $27(71)$ \\
\hline Age (y) & $61.0[42.8-74.1]$ & 57.1 [39.0-68.0] & $72.0[60.1-79.0]^{*}$ \\
\hline Weight (kg) & $77.5 \pm 15.0$ & $76.2 \pm 16.2$ & $79.3 \pm 13.0$ \\
\hline $\begin{array}{l}\text { Body mass } \\
\text { index }\left(\mathrm{kg} / \mathrm{m}^{2}\right)\end{array}$ & $27.1 \pm 4.3$ & $26.3 \pm 4.5$ & $28.3 \pm 3.8^{*}$ \\
\hline Hypertension & $60(65)$ & $26(47)$ & $34(90)^{*}$ \\
\hline Diabetes & 15 (16) & $5(9)$ & $10(26)^{*}$ \\
\hline CVD & $12(13)$ & $3(6)$ & $9(24)^{*}$ \\
\hline $\begin{array}{l}\text { eGFR } \\
\left(\mathrm{ml} / \mathrm{min} / 1.73 \mathrm{~m}^{2}\right)\end{array}$ & 43.0 [21.3-80.0] & 80.0 [64.5-96.0] & $22.0[10.0-35.3]^{\star}$ \\
\hline \multicolumn{4}{|l|}{ Medication } \\
\hline ACEi/ARB & $46(50)$ & $25(46)$ & $21(55)$ \\
\hline CCB & $34(37)$ & $13(24)$ & $21(55)^{\star}$ \\
\hline$\beta$-blockers & $20(21)$ & $10(18)$ & $10(26)$ \\
\hline ASA & $22(24)$ & $13(24)$ & $9(24)$ \\
\hline Statins & $38(41)$ & $14(26)$ & $24(63)^{\star}$ \\
\hline \multicolumn{4}{|l|}{ Lipid profile } \\
\hline $\begin{array}{l}\text { Total cholesterol } \\
(\mathrm{mmol} / \mathrm{L})\end{array}$ & $4.61 \pm 1.00$ & $4.43 \pm 0.86$ & $4.79 \pm 1.09$ \\
\hline $\mathrm{HDL}$ (mmol/L) & $1.37 \pm 0.39$ & $1.40 \pm 0.32$ & $1.34 \pm 0.45$ \\
\hline LDL (mmol/L) & $2.30 \pm 0.80$ & $2.29 \pm 0.84$ & $2.30 \pm 0.76$ \\
\hline $\mathrm{TG}(\mathrm{mmol} / \mathrm{L})$ & $2.02 \pm 1.16$ & $1.62 \pm 0.69$ & $2.44 \pm 1.38^{*}$ \\
\hline
\end{tabular}

Results are means $\pm S D, n(\%)$ or median (25th-75th percentiles). The $p$-value $<0.05$ is statistically significant.

$A C E i$, angiotensin-converting enzyme inhibitor; $A R B$, angiotensin receptor blockers; ASA, acetylsalicylic acid; CCB, calcium-channel blockers; CVD, cardiovascular disease; eGFR, estimated glomerular filtration rate from CKD-EPI equations; HDL, high-density lipoprotein; LDL, low-density lipoprotein; TG, triglycerides.

${ }^{*} A$ p-value of $<0.05$ than control group.

overall and in control and CKD subjects, respectively. Patients with CKD were older; had a higher BMI and triglyceride levels; had a higher prevalence of hypertension, cardiovascular disease; and diabetes; and were more frequently treated using calcium-channel blockers and statins. At baseline, there were significant correlations between eGFR, cf-PWV $(r=-0.37$, $p<0.001)$, AB-PWV ratio $(r=-0.38, p<0.001)$, AFPWV ratio $(r=-0.27, p=0.024)$, but not with cr-PWV $(r=-0.03, p=0.76)$, and fp-PWV $(r=-0.12, p=0.33)$ in this study population.

As shown in Table 2, after NTG administration, there was a slight, but statistically significant, decline in SBP, DBP, and PP. In the 56 subjects who had central pulse wave analysis before and after NTG administration, there was a reduction in AP@75 (8.8 \pm 7.4 vs. $3.6 \pm 5.6 \mathrm{mmHg} ; p<0.001)$ and AIx@75 (19.7 \pm 13.7 vs. $7.4 \pm 14.8 \% ; p<0.001)$. The end-systolic pressure decreased despite a reduction in ejection duration, but no changes were observed in the period. Finally, there was an increase in SEVR $(156.6 \pm 29.0$ vs. $163.4 \pm 31.3 \% ; p=0.004)$.
TABLE 2 | Nitroglycerin-induced changes in brachial pressures and regional arterial stiffness.

\begin{tabular}{|c|c|c|c|c|}
\hline & $\begin{array}{c}\text { Baseline } \\
n=93\end{array}$ & $\begin{array}{c}\text { NTG } \\
n=93\end{array}$ & $p$-value & $\begin{array}{c}p \text {-value } \\
\text { (adjusted } \\
\text { for DBP) }\end{array}$ \\
\hline \multicolumn{5}{|c|}{ Peripheral pressure parameters } \\
\hline $\mathrm{SBP}(\mathrm{mm} \mathrm{Hg})$ & $123.8 \pm 18.8$ & $119.2 \pm 17.2$ & $<0.001$ & \\
\hline $\mathrm{DBP}(\mathrm{mm} \mathrm{Hg})$ & $73.1 \pm 9.2$ & $71.9 \pm 10.0$ & 0.028 & \\
\hline $\mathrm{PP}(\mathrm{mm} \mathrm{Hg})$ & $50.7 \pm 16.1$ & $47.3 \pm 13.0$ & $<0.001$ & \\
\hline $\mathrm{MBP}(\mathrm{mm} \mathrm{Hg})$ & $92.2 \pm 11.5$ & $89.4 \pm 12.2$ & $<0.001$ & \\
\hline Heart rate (bpm) & $65.3 \pm 11.1$ & $66.2 \pm 10.6$ & 0.025 & \\
\hline \multicolumn{5}{|c|}{ Central pressure parameters $(n=56)$} \\
\hline $\mathrm{SBP}(\mathrm{mm} \mathrm{Hg})$ & $115.3 \pm 20.8$ & $108.3 \pm 16.6$ & $<0.001$ & \\
\hline $\mathrm{DBP}(\mathrm{mm} \mathrm{Hg})$ & $74.8 \pm 8.8$ & $73.5 \pm 9.0$ & 0.065 & \\
\hline $\mathrm{PP}(\mathrm{mm} \mathrm{Hg})$ & $41.7 \pm 18.4$ & $34.8 \pm 14.2$ & $<0.001$ & \\
\hline AP@75 (mm Hg) & $8.8 \pm 7.4$ & $3.6 \pm 5.6$ & $<0.001$ & \\
\hline Aix@75 (\%) & $19.7 \pm 13.7$ & $7.4 \pm 14.8$ & $<0.001$ & \\
\hline $\mathrm{P} 1(\mathrm{mmHg})$ & $29.2 \pm 9.1$ & $28.1 \pm 7.6$ & 0.160 & \\
\hline $\mathrm{T} 1$ (ms) & $105.0 \pm 9.5$ & $109.9 \pm 13.9$ & 0.009 & \\
\hline $\mathrm{TR}(\mathrm{ms})$ & $139.8 \pm 12.1$ & $146.1 \pm 14.9$ & $<0.001$ & \\
\hline T2 (ms) & $228.0 \pm 24.7$ & $211.2 \pm 27.7$ & $<0.001$ & \\
\hline $\begin{array}{l}\text { End-systolic pressure } \\
(\mathrm{mm} \mathrm{Hg})\end{array}$ & $104.1 \pm 16.7$ & $95.8 \pm 15.3$ & $<0.001$ & \\
\hline Ejection duration (ms) & $279.7 \pm 59.2$ & $267.5 \pm 67.0$ & $<0.001$ & \\
\hline Diastolic duration (ms) & $634.7 \pm 133.9$ & $644.1 \pm 137.0$ & 0.228 & \\
\hline Period (ms) & $965.1 \pm 156.3$ & $968.2 \pm 155.9$ & 0.696 & \\
\hline SEVR (\%) & $156.6 \pm 29.0$ & $163.4 \pm 31.3$ & 0.004 & \\
\hline PP amplification & $1.33 \pm 0.20$ & $1.47 \pm 0.22$ & $<0.001$ & \\
\hline \multicolumn{5}{|l|}{ Pulse wave velocities } \\
\hline cf-PWV (m/s) & $12.12 \pm 3.36$ & $12.14 \pm 3.55$ & 0.928 & 0.236 \\
\hline $\mathrm{cr}-\mathrm{PWV}(\mathrm{m} / \mathrm{s})$ & $9.51 \pm 1.81$ & $8.65 \pm 1.57$ & $<0.001$ & $<0.001$ \\
\hline $\mathrm{cp}-\mathrm{PWV}(\mathrm{m} / \mathrm{s})$ & $9.97 \pm 1.67$ & $9.02 \pm 1.24$ & $<0.001$ & $<0.001$ \\
\hline fp-PWV (m/s) & $9.71 \pm 1.89$ & $8.14 \pm 1.62$ & $<0.001$ & 0.032 \\
\hline $\begin{array}{l}\text { Aortic-brachial PWV } \\
\text { ratio }\end{array}$ & $1.30 \pm 0.39$ & $1.42 \pm 0.46$ & 0.001 & 0.001 \\
\hline $\begin{array}{l}\text { Aortic-femoral PWV } \\
\text { ratio }\end{array}$ & $1.38 \pm 0.47$ & $1.56 \pm 0.53$ & 0.001 & 0.005 \\
\hline
\end{tabular}

Results are means $\pm S D$. The $p$-values were obtained with paired-samples t-test and MBP-adjusted PWV were obtained with generalized estimating equations. The $p$-value $<0.05$ is statistically significant.

Aix@75, heart rate-adjusted augmentation index; AP@75, heart rate-adjusted augmented pressure; cp-PWW, carotid-dorsalis pedis pulse wave velocity; crPWV, carotid-radial pulse wave velocity; DBP, diastolic blood pressure; fp-PWV, femoral-dorsalis pedis pulse wave velocity; MBP, mean blood pressure; NTG, nitroglycerin; P1, first peak of pressure height; PP, pulse pressure; PP amplification, pulse pressure amplification; SBP, systolic blood pressure; SEVR, subendocardial viability ratio; T1, time of peak 1; T2, time of peak 2; TR, time to reflection of the reflected pressure wave.

\section{Impact of Nitroglycerin on Regional Stiffness and Arterial Stiffness Gradients}

As shown in Table 2 and Figures 1A-D, after NTG administration, there was no significant change in cf-PWV $(p=0.928)$, but there were significant reductions in both cr-PWV $(0.86 \pm 1.27 \mathrm{~m} / \mathrm{s} ; p<0.001)$ and fp-PWV $(1.12 \pm 1.74 \mathrm{~m} / \mathrm{s}$; $p<0.001)$. The results remained consistent even after adjustment for the small changes in DBP (Table 2). Consequently, as shown in Figures 1E,F, both AB-PWV and AF-PWV ratios increased 
A

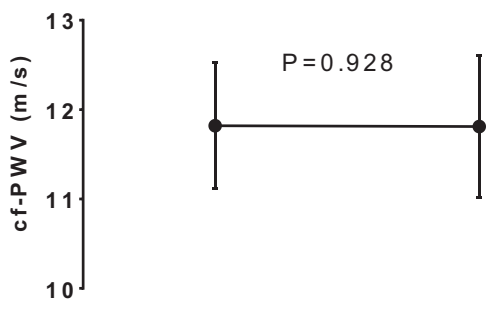

B

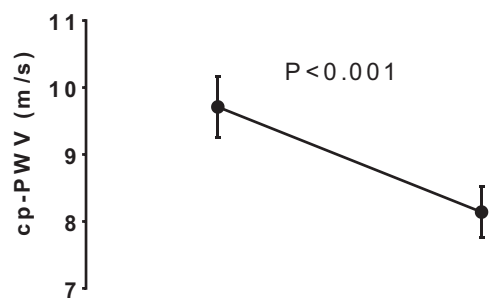

C

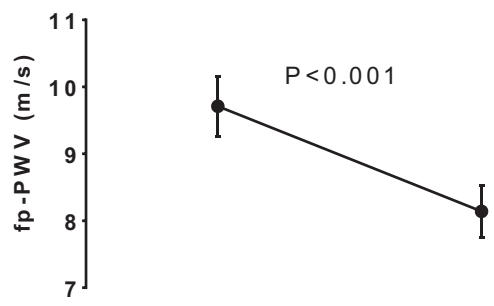

D

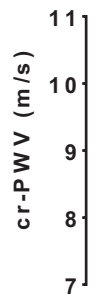

E

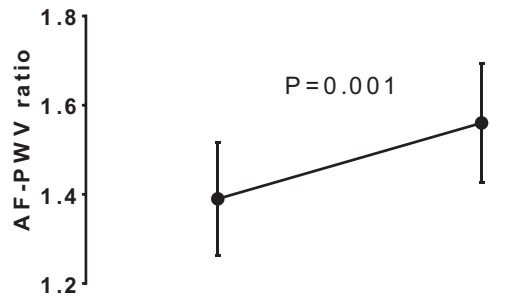

F

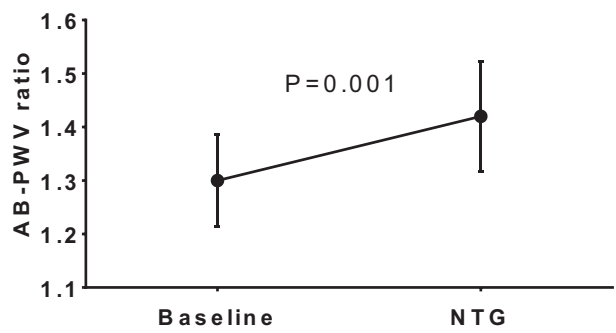

G

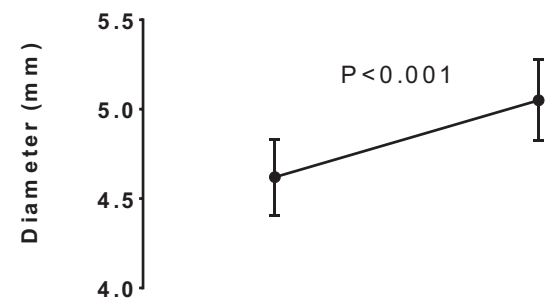

H

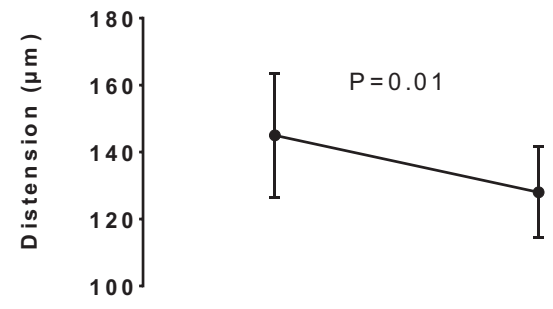

I

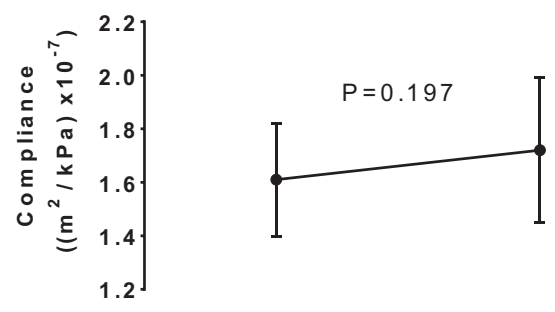

J

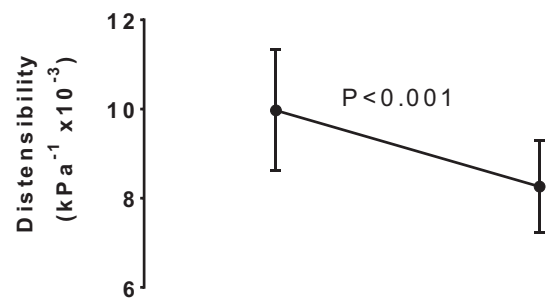

K

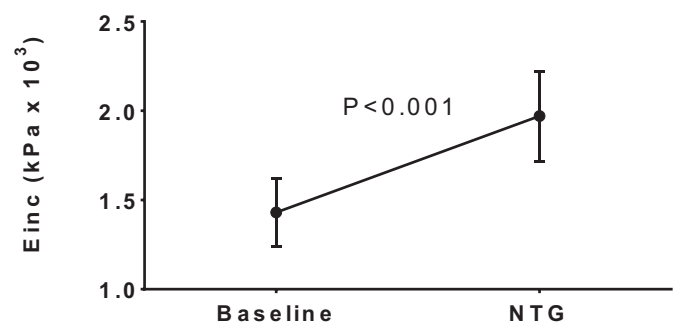

FIGURE 1 | Impact of NTG on regional stiffness, arterial stiffness gradients, and local brachial stiffness parameters. Panels (A-D) show the effect of nitroglycerin (NTG) on pulse wave velocity of various territories (PWV). The NTG had no effect on carotid-femoral PWV (cf-PWV) but significantly decreased carotid-pedis PWV (cp-PWV), femoral-pedis PWV (fp-PWV), and carotid-radial PWV (cr-PWV) leading to a corresponding increase in aortic-femoral PWV ratio [AF-PWV ratio (E)] and aortic-brachial PWV ratio [AB-PWV ratio (F)]. Changes in the brachial artery diameter (G), distention (H), compliance (I), distensibility (J), and incremental elastic modulus $\left[E_{\text {inc }},(\mathbf{K})\right]$ in response to NTG. 
significantly after NTG administration and remained significant even after adjustment for changes in DBP.

Table 3 shows the correlation matrix between changes in $A B-$ PWV and AF-PWV ratios and the TR and AP@75.

\section{Local Brachial Stiffness in Response to Nitroglycerin}

Table 4 describes the changes in the brachial artery diameter and stiffness parameters in response to NTG. As expected, after NTG, the brachial artery diameter $(4.62 \pm 0.80$ vs. $5.18 \pm 0.77 \mathrm{~mm} ; p<0.001)$ and the luminal crosssectional area $\left(17.2 \pm 5.9\right.$ vs. $\left.21.6 \pm 6.2 \mathrm{~mm}^{2} ; p<0.001\right)$ increased significantly. However, systolic-diastolic distention $(0.145 \pm 0.064$ vs. $0.128 \pm 0.047 \mathrm{~mm} ; p=0.010)$ and distensibility $\left(9.97 \pm 4.67\right.$ vs. $\left.8.26 \pm 3.56 \mathrm{kPa}^{-1} \times 10^{-3} ; p<0.001\right)$ decreased, incremental elastic modulus increased $(1.43 \pm 0.65$ vs. $\left.1.97 \pm 0.86 \mathrm{kPa}^{-1} \times 10^{3} ; p<0.001\right)$, without any significant changes in brachial artery compliance $(1.61 \pm 0.72$ vs. $\left.1.72 \pm 0.92 \mathrm{~m}^{2} \cdot \mathrm{kPa}^{-1} \times 10^{-7} ; p=0.197\right)$. These results are summarized in Figures 1G-K.

\section{Brachial Pulse Wave Velocity and Brachial Diameter}

To examine if the change in cr-PWV was related to brachial artery diameter, we performed a linear regression analysis and found that higher baseline cr-PWV and smaller baseline diameter were significant determinants in the decline of crPWV after NTG administration $\left(R^{2}=0.42\right.$; Table 5). Age, CKD, diabetes, and cardiovascular disease were not associated with changes in cr-PWV after NTG. We also examined and found no direct relationship between changes in cr-PWV and changes in brachial diameter or wall-to-lumen ratio in either absolute or relative terms.

\section{Vascular Response to Nitroglycerin by Chronic Kidney Disease Status}

Given the clinical heterogeneity of subjects with or without reduction in renal function, we examined if the vascular response to NTG could be affected by the assigned group. To examine this, we built an interaction term into the GEE equations and found no significant interaction between CKD status and changes in PWVs, PWV ratios, and brachial parameters after NTG.

TABLE 3 | Correlations between changes in arterial stiffness gradient and changes in wave reflection parameters.

\begin{tabular}{lccccc}
\hline & \multicolumn{2}{c}{$\boldsymbol{\Delta}$ AB-PWV ratio } & & \multicolumn{2}{c}{$\boldsymbol{\Delta}$ AF-PWV ratio } \\
\cline { 2 - 3 } \cline { 5 - 6 } & \multicolumn{1}{c}{$\boldsymbol{r}$} & $\boldsymbol{p}$-value & & $\boldsymbol{r}$ & $\boldsymbol{p}$-value \\
\hline$\Delta$ TR & 0.203 & 0.140 & & 0.289 & 0.042 \\
$\Delta$ AP@ 75 & -0.288 & 0.032 & & -0.467 & $<0.001$ \\
\hline
\end{tabular}

Correlation and $p$-values were obtained using Spearman rank bivariate correlation. The $p$-value $<0.05$ is statistically significant.

$A B-P W$, aortic-brachial pulse wave velocity; AF-PWW, aortic-femoral pulse wave velocity; TR, time to the reflection of the reflected pressure wave.
In addition, we examined and found no modifying effect of age (median 61 years old), sex, and established cardiovascular disease on the extent of studied vascular responses to NTG.

\section{DISCUSSION}

This study shows that acute administration of NTG reduces brachial and femoral PWVs without affecting aortic PWV, causing an increase in the AB-PWV and AF-PWV ratios. The delay in the timing of the reflected wave and the lowering of the amplitude of the reflection wave after NTG were best correlated with the changes in the AF-PWV ratio.

These observations are in line with previous studies showing that NTG reduces PWV in muscular peripheral arteries but has no effect in attenuating aortic PWV (Pauca et al., 2005; Yamamoto et al., 2017). These changes result in the attenuation or even the reversal of the arterial stiffness gradient. From a cardiac point of view, increasing the $\mathrm{AB}$ PWV and AF-PWV ratios and delaying the arrival and amplitude of the reflection wave in the ascending aorta may seem desirable, especially in the context of heart failure where nitrates and direct vasodilator (hydralazine) have been shown to improve left ventricular ejection fraction, peak oxygen consumption, and mortality (Cohn et al., 1991; Taylor et al., 2004). However, based on the wave propagation model, if there is less wave reflection in the ascending aorta, the forward pressure wave is less attenuated along the arterial tree, leading to a higher pulsatile pressure transmitted to the microcirculation. As stated earlier, given that the microcirculation is not structurally designed to accommodate a high pulse pressure, its chronic exposure to such mechanical stress may lead to microvascular dysfunction and end-organ damage. This is especially problematic for high flow/low resistance organs such as the brain and the kidneys (Mitchell, 2008). Indeed, in type 2 diabetic patients, Pierce et al. (2016) have observed that aortic-brachial stiffness gradient was associated with kidney function independent of aortic stiffness, implying that the arterial stiffness gradient may have a pathophysiological role in kidney disease. In addition, Mitchell et al. (2011) have shown that the loss of aortic-carotid impedance mismatch reduced the carotid reflection coefficient and facilitated the transmission of excessive flow pulsatility into the brain vasculature, which is presumably responsible for structural injuries to the brain microvasculature and the ensuing cognitive decline.

The clinical and biological determinants of a lower cr-PWV remain largely unknown and understudied. However, data are emerging that in certain conditions such as heart failure, kidney disease, or obesity in adolescents, the cr-PWV may be lower, hence contributing to the attenuation of the arterial stiffness gradient beyond the sole increase in aortic PWV (Mitchell et al., 2001; Zhu et al., 2007; Pierce et al., 2013, 2016; Utescu et al., 2013; Fortier et al., 2015; Agbaje et al., 2021). Moreover, the subjects of the African Americans of the atherosclerosis risk in communities (ARIC) study, tended to have a less favorable arterial stiffness gradient, not only due to higher cf-PWV but 
TABLE 4 | Brachial artery changes according to nitroglycerin administration.

\begin{tabular}{|c|c|c|c|c|c|}
\hline & $\begin{array}{c}\text { Baseline } \\
n=57\end{array}$ & $\begin{array}{c}\text { NTG } \\
n=57\end{array}$ & $p$-value & $\Delta$ NTG-baseline & $\begin{array}{c}p \text {-value (adjusted for } \\
\text { DBP) }\end{array}$ \\
\hline \multicolumn{6}{|l|}{ Brachial artery } \\
\hline Diameter (mm) & $4.62 \pm 0.80$ & $5.18 \pm 0.77$ & $<0.001$ & 0.57 (0.50 to 0.64$)$ & $<0.001$ \\
\hline IMT (mm) & $0.385 \pm 0.076$ & $0.346 \pm 0.072^{*}$ & - & $-0.037(-0.42$ to -0.33$)$ & - \\
\hline W/L ratio & $0.17 \pm 0.03$ & $0.14 \pm 0.03$ & $<0.001$ & $-0.03(-0.03$ to -0.02$)$ & $<0.001$ \\
\hline $\operatorname{LCSA}\left(\mathrm{mm}^{2}\right)$ & $17.2 \pm 5.9$ & $21.6 \pm 6.2$ & $<0.001$ & 4.33 (3.80 to 4.86$)$ & $<0.001$ \\
\hline WCSA $\left(\mathrm{mm}^{2}\right)$ & $6.13 \pm 1.92$ & - & - & - & - \\
\hline Distension (mm) & $0.145 \pm 0.064$ & $0.128 \pm 0.047$ & 0.010 & $-0.017(-0.029$ to -0.004$)$ & 0.012 \\
\hline Compliance $\left(\mathrm{m}^{2} \cdot \mathrm{kPa}^{-1} \times 10^{-7}\right)$ & $1.61 \pm 0.72$ & $1.72 \pm 0.92$ & 0.197 & $0.11(-0.06$ to 0.29$)$ & 0.254 \\
\hline Distensibility $\left(\mathrm{kPa}^{-1} \times 10^{-3}\right)$ & $9.97 \pm 4.67$ & $8.26 \pm 3.56$ & $<0.001$ & $-1.72(-2.69$ to -0.75$)$ & 0.006 \\
\hline Einc $\left(\mathrm{kPa} \times 10^{3}\right)$ & $1.43 \pm 0.65$ & $1.97 \pm 0.86$ & $<0.001$ & 0.55 (0.37 to 0.72$)$ & $<0.001$ \\
\hline
\end{tabular}

Results are means \pm SD or $(95 \% \mathrm{Cl})$. The $p$-values and mean changes in parameters with $95 \% \mathrm{Cl}$ were obtained using the paired-samples $t$-test, and MBP-adjusted $p$ values were obtained using generalized estimating equations. The $p$-value $<0.05$ is statistically significant.

$E_{\text {inc }}$, incremental elastic modulus; WCSA, mean wall cross-sectional area; IMT, intima media thickness; LCSA, Iuminal cross-sectional area; W/L ratio, wall-to-Iumen ratio. *Post-NTG IMT was not measured but calculated based on the conservation of wall cross-sectional area.

also because they presented with lower femoral-ankle PWV than Caucasians (Meyer et al., 2016).

These findings may suggest that the use of surrogate markers of arterial stiffness gradient may have a superior prognostic value above and beyond aortic stiffness. For example, in the dialysis population, we and others have shown that a higher $\mathrm{AB}-\mathrm{PWV}$ ratio is associated with the worst survival rate even after adjustments for potential confounding factors (Fortier et al., 2015; Bao et al., 2019). In addition, the AB-PWV ratio was associated with cardiovascular events after kidney transplantation $(p=0.045)$ but not cf-PWV $(p=0.501)$, and only the AB-PWV ratio has been associated with eGFR and albuminuria in a cohort of middle-aged women with increased cardiovascular risk (Laucyte-Cibulskiene et al., 2018, 2019). However, the superiority of the AB-PWV ratio over cf-PWV was not observed in patients at earlier stages of kidney disease (Bai et al., 2019). It is suggested that this is likely because the AB-PWV ratio increases significantly in patients once the eGFR decreases below $45 \mathrm{ml} / \mathrm{min} / 1.73 \mathrm{~m}^{2}$ (i.e., Stage $3 \mathrm{~B}$ and above) (Zanoli et al., 2018).

In the ARIC study population, a cross-sectional analysis showed that a low arterial stiffness gradient (femoral-ankle $\mathrm{PWV} / \mathrm{cf}-\mathrm{PWV}$ ) was associated with diabetes, coronary heart disease, heart failure, and stroke, whereas a high cf-PWV was only associated with hypertension (Stone et al., 2021). It should be mentioned that this is a relatively older cohort (age of 75 years) with a higher prevalence of hypertension (72\%) and diabetes

TABLE 5 | Determinants of change in carotid-radial PWV after NTG.

\begin{tabular}{lccc}
\hline Parameters & $\begin{array}{c}\text { Slope (95\% Confidence } \\
\text { interval) }\end{array}$ & $\boldsymbol{p}$-value & $\boldsymbol{R}^{\mathbf{2}}$ \\
\hline Final model & & & 0.421 \\
Constant & $-0.115(-2.315$ to 2.085$)$ & 0.917 & \\
Baseline $\mathrm{Cr}-\mathrm{PWV}(\mathrm{m} / \mathrm{s})$ & $-0.426(-0.614$ to -0.237$)$ & $<0.001$ & \\
Baseline brachial diameter $(\mathrm{mm})$ & $0.708(0.371$ to 1.046$)$ & $<0.001$ & \\
\hline
\end{tabular}

The final model of a forward conditional regression analysis where excluded variables (e.g., age, CKD, diabetes, and cardiovascular status) are not shown. The $p$-value $<0.05$ is statistically significant.
(29\%). In contrast, the AB-PWV ratio was not superior to cfPWV in predicting cardiovascular events in subjects from the Framingham Heart Study, a younger (mean age of 60 years) and healthier cohort ( $8 \%$ diabetes, 31\% receiving antihypertensive drugs) (Niiranen et al., 2017).

In this study, NTG resulted in vasodilation of the brachial artery $(12 \%)$, but this increase in diameter was accompanied by a lower degree of distention, showing no significant changes in the brachial artery compliance and even an increase in incremental elastic modulus. This increase in incremental elastic modulus could be explained by the transfer of pressure load from the vascular smooth muscle cells to the stiffer collagens of the extracellular matrix (Roach and Burton, 1957). Therefore, the reduction in cr-PWV could have potentially been explained by a decrease in a wall-to-lumen ratio according to the Moens-Korteweg equation $P W V=\sqrt{\frac{E i n c \cdot h}{2 r \cdot \rho}}$, where $h$ is the wall thickness and $r$ is the radius of the artery, and $\rho$ is the blood density. However, we found no statistical relationship between measured changes in cr-PWV and changes in either diameter or wall-to-lumen ratio. This discrepancy could be explained by several reasons. First, it could be that our spatial resolution was too limited for a reliable assessment of in vivo changes in wall-to-lumen ratio. Second, it could be related to the violation of basic assumptions underlying the equation, i.e., that the arterial wall is isotropic and behaves in an isovolumetric manner in response to pulse pressure (Gosling and Budge, 2003). In addition, since in vivo arterial diameter at zero transmural pressure could not be measured, Hooke's law was applied for the determination of $\mathrm{E}_{\mathrm{inc}}$ (Roark and Young, 1989), therefore implying geometrical assumptions that the arterial segments were considered as uniform thick-walled tubes. However, this assumption does not take into account any geometrical variation along the arterial wall. Finally, this discrepancy can be explained by the heterogeneity of the arterial tree between carotid and radial artery both in terms of diameter, arterial wall thickness, and composition. As such, the measured PWV over an arterial path is the summation of the local vascular responses along with the studied segments (i.e., ignoring potential heterogeneity of the vascular response). This possibility is further supported 
by the results from the PWV between the carotid and dorsal pedis artery, which declined after NTG, but this reduction was solely due to the reduction over the vascular bed below the femoral artery.

Besides arterial stiffness gradient, this study underlines the importance of proper assessment of regional vascular stiffness and its response to therapy. Given the nature of translational medicine, we have been eager to take the arterial stiffness assessments out of specialized laboratories and into the largescale epidemiological studies and clinical practice through simplification of arterial stiffness assessment. For example, our study clearly shows that PWV from the carotid to the dorsal pedis artery declined significantly after NTG, but upon closer look, we saw that this decline was related to the reduction in PWV from the femoral to the dorsal pedis segment and not related to aortic PWV per se. This observation is in line with the findings of Yamamoto et al. (2017) using cardio-ankle vascular index (CAVI) and heart-thigh and thigh-ankle B-stiffness indices.

This study has several strengths and limitations. First, it involves a large number of subjects with a detailed evaluation of various regional pulse wave velocities, central pulse wave profile, and brachial stiffness parameters. Second, given the heterogeneity of the subjects, we were able to perform various sensitivity analyses showing the robustness of our findings. Indeed, older subjects may have a more hypotensive response to NTG (Cahalan et al., 1992). However, even if our CKD patients were older and had several other heterogeneities, it did not modify the vascular responses in this study. There are also limitations that should be mentioned. First, we did not examine the smaller arteries of the vascular bed through the assessment of radial-digital PWV (Obeid et al., 2021). Second, it would have also been interesting to assess the local distensibility of both carotid and femoral arteries. However, given that we only had a 5 -min window to measure all PWVs in triplicates and brachial distensibility, the addition of local carotid and femoral distensibilities would have pushed us outside the desired window, and besides technical limitations, it would have added uncertainties regarding the potential fading effect of NTG. Third, our interpretation of results is based on the pulse wave propagation model, which does not consider the reservoir function of the arterial tree (Wang et al., 2003; Tyberg et al., 2009; Parker et al., 2012; Mynard and Smolich, 2014). In addition, there are two limitations regarding the use of augmentation index (AIx) as a marker of wave reflection, namely, the reliability of the central AIx estimated via transfer function from the radial pressure waveform and the validity of central AIx as a measure of wave reflection (Chen et al., 1996; Millasseau et al., 2003; Segers et al., 2005; Miyashita, 2012; Hughes et al., 2013; Heusinkveld et al., 2019). Indeed, the central AIx derived from the generalized transfer function seems to correlate more strongly with the AIx of the original peripheral waveform rather than the central pressure waveform (Segers et al., 2005). Furthermore, the AIx and the related arrival time may be influenced by other factors, such as myocardial contractility, and hence may not be a proper measure of wave reflection (Hughes et al., 2013; Heusinkveld et al., 2019). Finally, our results are limited to the vascular response to NTG in an acute setting and do not imply similar effects would necessarily occur in a chronic setting.

\section{CONCLUSION}

While there are statistical associations between markers of arterial stiffness gradient and certain clinical conditions in both crosssectional and longitudinal studies, there has not been a systematic focus on the impact of pharmacological interventions on the arterial stiffness gradient. In light of recent evidence supporting the potential usefulness of arterial stiffness gradient assessment, and our present data showing an alteration in arterial stiffness gradient in response to NTG, we would like to underline the importance of assessing regional PWV in order to develop an integrated approach into the arterial tree and its response to therapy. As such, these results are hypothesis generating and their clinical and pharmacological usefulness should be assessed in properly designed studies.

\section{DATA AVAILABILITY STATEMENT}

The raw data supporting the conclusions of this article will be made available by the authors, without undue reservation.

\section{ETHICS STATEMENT}

The studies involving human participants were reviewed and approved by Comité d'Éthique de la Recherche du CHU de Québec. The patients/participants provided their written informed consent to participate in this study.

\section{AUTHOR CONTRIBUTIONS}

MA and CF provided the conception and design of the study. $\mathrm{CF}, \mathrm{C}-\mathrm{AG}, \mathrm{MP}, \mathrm{HO}, \mathrm{NC}, \mathrm{KD}$, and $\mathrm{MA}$ recruited participants and collected data. $\mathrm{CF}, \mathrm{C}-\mathrm{AG}$, and $\mathrm{MA}$ performed the statistical analysis and wrote the manuscript. All authors contributed to manuscript revision, read, and approved the submitted version.

\section{FUNDING}

This project was supported by a grant from Canadian Institutes of Health Research (CIHR-201903PJT - 419835). C-AG and MP were supported by scholarships from Fonds de Recherche du Québec-Santé (FRQ-S), CIHR, and Université Laval. HO was supported by a postdoctoral scholarship from the Société Québécoise d'Hypertension Artérielle and from Fondation de l'Université Laval. RG was supported by a FRQ-S clinicianscientist scholarship.

\section{ACKNOWLEDGMENTS}

We are grateful to the clinic personnel of hypertension and CKD and the study subjects for their kind contribution and precious collaboration. 


\section{REFERENCES}

Agbaje, A. O., Barker, A. R., and Tuomainen, T.-P. (2021). Cardiorespiratory fitness, fat mass, and cardiometabolic health with endothelial function, arterial elasticity, and stiffness. Med. Sci. Sports Exerc. doi: 10.1249/MSS. 0000000000002757

Bai, Q., Su, C.-Y., Zhang, A.-H., Wang, T., and Tang, W. (2019). Loss of the normal gradient in arterial compliance and outcomes of chronic kidney disease patients. Cardiorenal. Med. 9, 297-307. doi: 10.1159/000500479

Bao, W., Wang, F., and Tang, W. (2019). Aortic-brachial stiffness mismatch and mortality in peritoneal dialysis patients. Kidney Blood Press Res. 44, 123-132. doi: $10.1159 / 000498876$

Blacher, J., Asmar, R., Djane, S., London, G. M., and Safar, M. E. (1999a). Aortic pulse wave velocity as a marker of cardiovascular risk in hypertensive patients. Hypertension 33, 1111-1117. doi: 10.1161/01.hyp.33.5.1111

Blacher, J., Guerin, A. P., Pannier, B., Marchais, S. J., Safar, M. E., and London, G. M. (1999b). Impact of aortic stiffness on survival in end-stage renal disease. Circulation 99, 2434-2439. doi: 10.1161/01.cir.99.18.2434

Bortolotto, L. A., Hanon, O., Franconi, G., Boutouyrie, P., Legrain, S., and Girerd, X. (1999). The aging process modifies the distensibility of elastic but not muscular arteries. Hypertension 34, 889-892. doi: 10.1161/01.hyp.34.4.889

Cahalan, M. K., Hashimoto, Y., Aizawa, K., Verotta, D., Ionescu, P., Balea, M., et al. (1992). Elderly, conscious patients have an accentuated hypotensive response to nitroglycerin. Anesthesiology 77, 646-655. doi: 10.1097/00000542-19921000000006

Chen, C. H., Ting, C. T., Nussbacher, A., Nevo, E., Kass, D. A., Pak, P., et al. (1996). Validation of carotid artery tonometry as a means of estimating augmentation index of ascending aortic pressure. Hypertension 27, 168-175. doi: 10.1161/01. hyp.27.2.168

Cohn, J. N., Johnson, G., Ziesche, S., Cobb, F., Francis, G., Tristani, F., et al. (1991). A comparison of enalapril with hydralazine-isosorbide dinitrate in the treatment of chronic congestive heart failure. N. Engl. J. Med. 325, 303-310. doi: 10.1056/NEJM199108013250502

Cruickshank, K., Riste, L., Anderson, S. G., Wright, J. S., Dunn, G., and Gosling, R. G. (2002). Aortic pulse-wave velocity and its relationship to mortality in diabetes and glucose intolerance: an integrated index of vascular function? Circulation 106, 2085-2090. doi: 10.1161/01.cir.0000033824.02722.f7

Fortier, C., and Agharazii, M. (2016). Arterial stiffness gradient. Pulse Basel Switz. 3, 159-166. doi: 10.1159/000438852

Fortier, C., Mac-Way, F., Desmeules, S., Marquis, K., De Serres, S. A., Lebel, M., et al. (2015). Aortic-brachial stiffness mismatch and mortality in dialysis population. Hypertension 65, 378-384. doi: 10.1161/HYPERTENSIONAHA. 114.04587

Gosling, R. G., and Budge, M. M. (2003). Terminology for describing the elastic behavior of arteries. Hypertension 41, 1180-1182. doi: 10.1161/01.HYP. $0000072271.36866 .2 \mathrm{~A}$

Hashimoto, J., and Ito, S. (2013). Aortic stiffness determines diastolic blood flow reversal in the descending thoracic aorta: potential implication for retrograde embolic stroke in hypertension. Hypertension 62, 542-549. doi: 10.1161/ HYPERTENSIONAHA.113.01318

Heusinkveld, M. H. G., Delhaas, T., Lumens, J., Huberts, W., Spronck, B., Hughes, A. D., et al. (2019). Augmentation index is not a proxy for wave reflection magnitude: mechanistic analysis using a computational model. J. Appl. Physiol. Bethesda Md. 127, 491-500. doi: 10.1152/japplphysiol.00769.2018

Hughes, A. D., Park, C., Davies, J., Francis, D., McG Thom, S. A., Mayet, J., et al. (2013). Limitations of augmentation index in the assessment of wave reflection in normotensive healthy individuals. PLoS One 8:e59371. doi: 10.1371/journal. pone.0059371

Karras, A., Haymann, J.-P., Bozec, E., Metzger, M., Jacquot, C., Maruani, G., et al. (2012). Large artery stiffening and remodeling are independently associated with all-cause mortality and cardiovascular events in chronic kidney disease. Hypertension 60, 1451-1457. doi: 10.1161/HYPERTENSIONAHA.112.197210

Kidney Disease Improving Global Outcomes [KDIGO] (2013). Chapter 1: definition and classification of CKD. Kidney Int. Suppl. 3, 19-62. doi: 10.1038/ kisup. 2012.64

Laucyte-Cibulskiene, A., Boreikaite, E., Aucina, G., Gudynaite, M., Rudminiene, I., Anisko, S., et al. (2018). Usefulness of pretransplant aortic arch calcification evaluation for kidney transplant outcome prediction in one year follow-up. Ren. Fail. 40, 201-208. doi: 10.1080/0886022X.2018.1455588

Laucyte-Cibulskiene, A., Vickiene, A., Ryliskyte, L., Badariene, J., Rimsevicius, L., and Miglinas, M. (2019). Should we calculate arterial stiffness gradient in middle-aged women with increased cardiovascular risk? Blood Press 28, 199-205. doi: 10.1080/08037051.2019.1591921

Laurent, S., Boutouyrie, P., Asmar, R., Gautier, I., Laloux, B., Guize, L., et al. (2001). Aortic stiffness is an independent predictor of all-cause and cardiovascular mortality in hypertensive patients. Hypertension 37, 1236-1241. doi: 10.1161/ 01.hyp.37.5.1236

Laurent, S., Cockcroft, J., Van Bortel, L., Boutouyrie, P., Giannattasio, C., Hayoz, D., et al. (2006). Expert consensus document on arterial stiffness: methodological issues and clinical applications. Eur. Heart J. 27, 2588-2605. doi: 10.1093/ eurheartj/ehl254

Laurent, S., Girerd, X., Mourad, J. J., Lacolley, P., Beck, L., Boutouyrie, P., et al. (1994). Elastic modulus of the radial artery wall material is not increased in patients with essential hypertension. Arterioscler. Thromb. J. Vasc. Biol. 14, 1223-1231. doi: 10.1161/01.ATV.14.7.1223

Levey, A. S., Stevens, L. A., Schmid, C. H., Zhang, Y. L., Castro, A. F., Feldman, H. I., et al. (2009). A new equation to estimate glomerular filtration rate. Ann. Intern. Med. 150, 604-612. doi: 10.7326/0003-4819-150-9-200905050-00006

Meyer, M. L., Tanaka, H., Palta, P., Cheng, S., Gouskova, N., Aguilar, D., et al. (2016). Correlates of segmental pulse wave velocity in older adults: the Atherosclerosis Risk in Communities (ARIC) study. Am. J. Hypertens. 29, 114-122. doi: 10.1093/ajh/hpv079

Millasseau, S. C., Patel, S. J., Redwood, S. R., Ritter, J. M., and Chowienczyk, P. J. (2003). Pressure wave reflection assessed from the peripheral pulse: is a transfer function necessary? Hypertension 41, 1016-1020. doi: 10.1161/01.HYP. 0000057574.64076.A5

Mitchell, G. F. (2008). Effects of central arterial aging on the structure and function of the peripheral vasculature: implications for end-organ damage. J. Appl. Physiol. Bethesda Md. 105, 1652-1660. doi: 10.1152/japplphysiol.90549.2008

Mitchell, G. F., Tardif, J. C., Arnold, J. M., Marchiori, G., O’Brien, T. X., Dunlap, M. E., et al. (2001). Pulsatile hemodynamics in congestive heart failure. Hypertension 38, 1433-1439. doi: 10.1161/hy1201.098298

Mitchell, G. F., van Buchem, M. A., Sigurdsson, S., Gotal, J. D., Jonsdottir, M. K., Kjartansson, Ó, et al. (2011). Arterial stiffness, pressure and flow pulsatility and brain structure and function: the Age, Gene/Environment SusceptibilityReykjavik study. Brain J. Neurol. 134, 3398-3407. doi: 10.1093/brain/awr253

Miyashita, H. (2012). Clinical assessment of central blood pressure. Curr. Hypertens. Rev. 8, 80-90. doi: 10.2174/157340212800840708

Mynard, J. P., and Smolich, J. J. (2014). Wave potential and the one-dimensional windkessel as a wave-based paradigm of diastolic arterial hemodynamics. Am. J. Physiol. Heart Circ. Physiol. 307, H307-H318. doi: 10.1152/ajpheart. 00293.2014

Niiranen, T. J., Kalesan, B., Larson, M. G., Hamburg, N. M., Benjamin, E. J., Mitchell, G. F., et al. (2017). Aortic-brachial arterial stiffness gradient and cardiovascular risk in the community: the framingham heart study. Hypertension 69, 1022-1028. doi: 10.1161/HYPERTENSIONAHA.116. 08917

Obeid, H., Fortier, C., Garneau, C.-A., Pare, M., Boutouyrie, P., Bruno, R. M., et al. (2021). Radial-digital pulse wave velocity: a noninvasive method for assessing stiffness of small conduit arteries. Am. J. Physiol. Heart Circ. Physiol. 320, H1361-H1369. doi: 10.1152/ajpheart.00551.2020

Padhy, S., Kar, P., and Ramachandran, G. (2017). Prediction of Major Adverse Cardiovascular and Cerebrovascular Events (MACCE) after thoracic surgerythe role of estimated GFR. J. Clin. Diagn. Res. JCDR 11, UC13-UC16. doi: 10.7860/JCDR/2017/27428.10616

Parker, K. H., Alastruey, J., and Stan, G.-B. (2012). Arterial reservoir-excess pressure and ventricular work. Med. Biol. Eng. Comput. 50, 419-424. doi: 10. 1007/s11517-012-0872-1

Pauca, A. L., Kon, N. D., and O’Rourke, M. F. (2005). Benefit of glyceryl trinitrate on arterial stiffness is directly due to effects on peripheral arteries. Heart $\mathrm{Br}$. Card. Soc. 91, 1428-1432. doi: 10.1136/hrt.2004.057356

Pierce, G. L., Pajaniappan, M., DiPietro, A., Darracott-Woei-A-Sack, K., and Kapuku, G. K. (2016). Abnormal central pulsatile hemodynamics in adolescents with obesity: higher aortic forward pressure wave amplitude is independently 
associated with greater left ventricular mass. Hypertension 68, 1200-1207. doi: 10.1161/HYPERTENSIONAHA.116.07918

Pierce, G. L., Zhu, H., Darracott, K., Edet, I., Bhagatwala, J., Huang, Y., et al. (2013). Arterial stiffness and pulse-pressure amplification in overweight/obese AfricanAmerican adolescents: relation with higher systolic and pulse pressure. Am. J. Hypertens. 26, 20-26. doi: 10.1093/ajh/hps014

Roach, M. R., and Burton, A. C. (1957). The reason for the shape of the distensibility curves of arteries. Can. J. Biochem. Physiol. 35, 681-690.

Roark, R. J., and Young, W. C. (1989). Roark's Formulas for Stress and Strain, 6th Edn. New York, NY: McGraw-Hill.

Segers, P., Rietzschel, E., Heireman, S., De Buyzere, M., Gillebert, T., Verdonck, P., et al. (2005). Carotid tonometry versus synthesized aorta pressure waves for the estimation of central systolic blood pressure and augmentation index. Am. J. Hypertens. 18, 1168-1173. doi: 10.1016/j.amjhyper.2005.04.005

Spronck, B., Tan, I., Reesink, K. D., Georgevsky, D., Delhaas, T., Avolio, A. P., et al. (2021). Heart rate and blood pressure dependence of aortic distensibility in rats: comparison of measured and calculated pulse wave velocity. J. Hypertens. 39, 117-126. doi: 10.1097/HJH.0000000000002608

Stone, K., Fryer, S., Meyer, M. L., Kucharska-Newton, A., Faulkner, J., Zieff, G., et al. (2021). The aortic-femoral arterial stiffness gradient: an atherosclerosis risk in communities (ARIC) study. J. Hypertens. 39, 1370-1377. doi: 10.1097/ HJH.0000000000002808

Tarumi, T., Shah, F., Tanaka, H., and Haley, A. P. (2011). Association between central elastic artery stiffness and cerebral perfusion in deep subcortical gray and white matter. Am. J. Hypertens. 24, 1108-1113. doi: 10.1038/ajh.2011.101

Taylor, A. L., Ziesche, S., Yancy, C., Carson, P., D’Agostino, R., Ferdinand, K., et al. (2004). Combination of isosorbide dinitrate and hydralazine in blacks with heart failure. N. Engl. J. Med. 351, 2049-2057. doi: 10.1056/NEJMoa042934

Tyberg, J. V., Davies, J. E., Wang, Z., Whitelaw, W. A., Flewitt, J. A., Shrive, N. G., et al. (2009). Wave intensity analysis and the development of the reservoirwave approach. Med. Biol. Eng. Comput. 47, 221-232. doi: 10.1007/s11517-0080430-z

Utescu, M. S., Couture, V., Mac-Way, F., De Serres, S. A., Marquis, K., Larivière, R., et al. (2013). Determinants of progression of aortic stiffness in hemodialysis patients: a prospective longitudinal study. Hypertension 62, 154-160. doi: 10 . 1161/HYPERTENSIONAHA.113.01200
Wang, J.-J., O’Brien, A. B., Shrive, N. G., Parker, K. H., and Tyberg, J. V. (2003). Time-domain representation of ventricular-arterial coupling as a windkessel and wave system. Am. J. Physiol. Heart Circ. Physiol. 284, H1358-H1368. doi: 10.1152/ajpheart.00175.2002

Wilkinson, I. B., Qasem, A., McEniery, C. M., Webb, D. J., Avolio, A. P., and Cockcroft, J. R. (2002). Nitric oxide regulates local arterial distensibility in vivo. Circulation 105, 213-217. doi: 10.1161/hc0202.101970

Yamamoto, T., Shimizu, K., Takahashi, M., Tatsuno, I., and Shirai, K. (2017). The effect of nitroglycerin on arterial stiffness of the aorta and the femoral-tibial arteries. J. Atheroscler. Thromb. 24, 1048-1057. doi: 10.5551/jat. 38646

Zanoli, L., Lentini, P., Boutouyrie, P., Fatuzzo, P., Granata, A., Corrao, S., et al. (2018). Pulse wave velocity differs between ulcerative colitis and chronic kidney disease. Eur. J. Intern. Med. 47, 36-42. doi: 10.1016/j.ejim.2017. 08.020

Zhu, H., Yan, W., Ge, D., Treiber, F. A., Harshfield, G. A., Kapuku, G., et al. (2007). Cardiovascular characteristics in American youth with prehypertension. Am. J. Hypertens. 20, 1051-1057. doi: 10.1016/j.amjhyper.2007.05.009

Conflict of Interest: The authors declare that the research was conducted in the absence of any commercial or financial relationships that could be construed as a potential conflict of interest.

Publisher's Note: All claims expressed in this article are solely those of the authors and do not necessarily represent those of their affiliated organizations, or those of the publisher, the editors and the reviewers. Any product that may be evaluated in this article, or claim that may be made by its manufacturer, is not guaranteed or endorsed by the publisher.

Copyright (c) 2021 Fortier, Garneau, Paré, Obeid, Côté, Duval, Goupil and Agharazii. This is an open-access article distributed under the terms of the Creative Commons Attribution License (CC BY). The use, distribution or reproduction in other forums is permitted, provided the original author(s) and the copyright owner(s) are credited and that the original publication in this journal is cited, in accordance with accepted academic practice. No use, distribution or reproduction is permitted which does not comply with these terms. 